\title{
Numerical simulation and statistical analysis of random response of the landing gear at taxiing
}

\author{
Wu Weiguo ${ }^{1,2, a^{*}}$, Jia Teng ${ }^{1, b}$ Xiong Furui ${ }^{1, c}$ \\ ${ }^{1}$ Tianjin University, School of Mechanical Engineering, Tianjin 300072, China \\ ${ }^{2}$ Military Transportation Research Institute of the General Logistics Department, Tianjin 300161, China \\ awwg223@126.com, bjiateng723@163.com, cfineday575@sohu.com
}

Keywords: landing gear; runway unevenness; random simulation; random response; statistical analysis.

Abstract. Due to the runway unevenness, random vibration will occur of aircraft landing gear during impacting and taxiing phase. In view of the simplified two degrees of freedom model of landing gear, the nonlinear dynamic equation of the system can be established. By using the military specification's formula of runway unevenness, large number of unevenness time series can be generated via the harmonic superposition method. The 10000 samples whose confidence interval width is smaller and whose simulated time is shorter are taken as the unevenness test samples. With the runway unevenness of 10000 samples as input, solving the nonlinear dynamic equation of the system yields a large number of samples of displacement response. Through statistical analysis of response samples in the random data analysis method, some statistical information such as mean, mean square, auto-correlation function and PSD of the displacements can be obtained. Results show that through the analysis of the PDF of displacements, the distribution of displacement response is non-Gaussian.

\section{Introduction}

The research of landing gear is important for the design and analysis of aircraft ${ }^{[1]}$. The landing gear is not involved in sustaining the weight of aircraft in the flying. The safety of aircraft relies on the landing gear in the takeoff and landing stages. In the designing process of landing gear, the analysis of its dynamic performance is as important as the static stress analysis.

While the aircraft is taxiing on the runway, the random vibrations of landing gear are caused by runway unevenness. The adverse effects of random vibrations are as follows: (1) It has an impact on the fatigue life of the aircraft landing gear; (2) The vibration will affect the pilot's normal work; (3) For the large aircraft, it will affect the comfort and safety of passengers. To solve this problem, foreign scholars studied the random vibrations of landing gear with the mature mehtod in the early period. The first method is a deterministic numerical method by which the equations including the nonlinear force of landing gear are solved. The method is only for a specific track, with its measured data as input, which the vibration impact can be calculated from runway unevenness.

The second method is frequency-domain analysis method, which the runway excitation is expressed by the stationary random process ${ }^{[2]}$. Frequency domain analysis is premised on the system linearization. The result was acquired with the transfer function and input excitation. However, a weak non-linear impact was considered in this method. The damping of buffer was equivalent linearization by Krik CL, with the linear equivalent damping coefficient by using the principle of energy equivalent ${ }^{[3]}$. The aircraft taxiing loads were studied by researchers, and it has been combined with the control system for analyzing the status of landing gear on takeoff and landing roll stages. Liu $\mathrm{Li}^{[4]}$ studied two degrees of freedom model of the landing gear's taxiing loads with the frequency domain analysis and analyzed the impact on the taxiing dynamic response from the buffer system parameters; Nie Hong ${ }^{[5]}$ studied the dynamic characteristics of the aircraft landing gear with variable taxiing speed; Liu Rui-chen ${ }^{[6]}$ described the basic method for the determination of random data track unevenness based on the concept of the power spectrum, meanwhile he analyzed the runway unevenness power spectrum curve fitting and the landing gear's taxiing dynamic response. Li Si-zheng ${ }^{[1]}$ studied the taxiing dynamic response of multiple wheels and multiple landing gears. 
In short, the current frequency domain analysis method is relatively common. However, in the process of structural parameters linearization, some important information will be ignored. For the strong nonlinear system, sometimes it can not be linearized. The result is accurate but time-consuming with the data measured. The application of stochastic simulation method can solve the problem, which the computer can simulate the runway unevenness data.

\section{The landing gear model}

\section{The calculation model.}

According to the motion characteristics of the landing gear structure, the quality of the landing gear structure needs to be divided into two lumped masses ${ }^{[4,5,7]}:$ (1) the quality sustained by air spring. The portion mass sustained by the air spring, including the fuselage, wings, bumper outer tube and so on in the landing gear stationary state; (2) the quality not sustained by air spring. The part of the quality sustained by the ground, including the buffer rod, brakes, wheels and so on in the landing gear stationary state.

\section{The dynamic model.}

The simplified landing gear model is depicted in Figure 1. Dynamic equations ${ }^{[\underline{[}]}$ of two degrees of freedom landing gear are given in the equation (1).

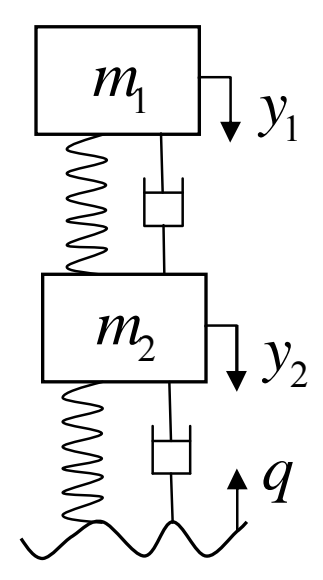

$$
\begin{aligned}
& m_{1} \propto=m_{1} g-F_{a}-F_{l}-f \\
& m_{2}=m_{2} g+F_{a}+F_{l}+f-F_{t} \\
& F_{a}=P_{0} A\left[\frac{V_{0}}{V_{0}-A\left(y_{s}+y_{s}^{*}\right)}\right]^{\gamma} \\
& F_{l}=\frac{\left.\rho A^{3}\right|_{s}\left|\Phi_{s}\right|}{2 \varsigma^{2} A_{0}^{2}}
\end{aligned}
$$

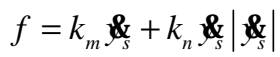

$$
\begin{aligned}
& F_{t}=k_{t}\left(y_{2}+y_{2}{ }^{*}+q\right)+c_{t}(\phi) \\
& y_{2}{ }^{*}=\frac{\left(m_{1}+m_{2}\right) g}{k_{t}} \\
& y_{1}^{*}=y_{2}^{*}+\frac{V_{0}}{A}\left[1-\left(\frac{P_{0} A}{m_{1} g}\right)^{\frac{1}{\gamma}}\right]
\end{aligned}
$$

Fig. 1 Simplified model

where, $F_{a}, F_{l}, f, F_{t}$ are the gas spring force, damping force, friction and tire reaction force respectively. The sum of the gas spring force, damping force and friction is called the strut force. Denote $F_{s}$ as the strut force. Obviously, we have the relationship $F_{s}=F_{a}+F_{l}+f$. The strut force is the macroscopic indication of the gear fuselage interaction, which is an important cause of fuselage fatigue and damage. The upper mass and lower mass are denoted as $m_{1}$ and $m_{2}$. Coordinate transformation is performed to put the system's equilibrium to the origin, i.e. $y_{1}=y_{1} / y_{1}{ }^{*}$, and $y_{2}=9 \% y_{2}{ }^{*}$, where $y_{1}^{*}, y_{2}{ }^{*}$ are the static positions of the upper and lower mass given in equation (6) and equation (7). Let $y_{s}=y_{1}-y_{2}$ be the landing gear piston stroke and be the stroke velocity. $P_{0}, V_{0}, A$ are $\gamma$ the initial pressure, initial volume and cross section area of the upper gas chamber. $\rho, \zeta$ and $A_{0}$ are the damping oil density, orifice discharge coefficient and orifice area. $k_{m}$ and $k_{n}$ are the empirical parameter to model the friction. $q$ is the runway unevenness. The tire is modeled by a linear spring damper with $k_{t}$ and $c_{t}$ as the stiffness and damping coefficient. 


\section{The stochastic simulation of runway unevenness}

\section{The power spectrum of runway unevenness}

According to the measured results, the literatures ${ }^{[9]}{ }^{[10]}$ describe the road surface spectrum curve fitting experience expressions,

$$
\Phi(\Omega)=\frac{C}{\Omega^{A}}
$$

where $\Omega$ is the space circular frequency, $\Omega=2 \pi f / v, f$ is temporal frequency. For most aircrafts, $f=0.5 \sim 35 \mathrm{~Hz}, v$ is taxiing speed. $A$ and $C$ represent the runway characteristic coefficient. When the taxiing speed is given, the power spectral density expression about temporal frequency is given in equation $(9)^{[1]}$.

$$
S(f)=\frac{\Phi(\Omega)}{v}=\frac{C v^{A-1}}{(2 \pi)^{A} f^{A}}
$$

\section{The harmony superposition method}

Random signal can be decomposed into a series of sine waves with different frequencies and amplitudes by discrete Fourier transform. The harmonic superposition method is a discrete numerical simulation method runway ${ }^{[11]}$. Considering the runway unevenness as a stationary random process, runway unevenness variance can be expressed as follows:

$$
\sigma_{q}^{2}=\int_{f_{1}}^{f_{2}} \Phi(f) d f
$$

The Interval $\left(f_{1}, f_{2}\right)$ is divided into $N$ small sections. The width of small section $i$ is $\Delta f_{i}$. The $\Phi\left(f_{i}\right)$ is instead of the power spectrum density $\Phi\left(f_{\text {mid }_{-} i}\right)$, where the center frequency is $f_{\text {mid }_{-} i}$.

$$
\sigma_{q}^{2} \approx \sum_{i=1}^{N} \Phi\left(f_{\text {mid_i }}\right) \Delta f_{i}
$$

When the taxiing speed $(v)$ is constant, the space sine wave function of runway unevenness can be expressed as follows, where the frequency is $f_{\text {mid } \_}(i=1,2,3, \mathrm{~K}, N)$ and the standard deviation is

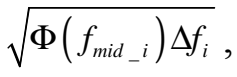

$$
\sqrt{2 \Phi\left(f_{\text {mid_i }}\right) \Delta f_{i}} \sin \left(2 \pi \frac{f_{\text {mid_i } i}}{v} x+\theta_{i}\right)
$$

When all the small sections of the sine function are added together, a runway unevenness space expression can be acquired,

$$
q(x)=\sum_{i=1}^{N} \sqrt{2 \Phi\left(f_{\text {mid_i }_{-}}\right) \Delta f_{i}} \sin \left(2 \pi \frac{f_{\text {mid_i }} i}{v} x+\theta_{i}\right)
$$

where $\theta_{i}$ is the independent random variable between the interval $[0,2 \pi]$, which is uniform distribution. $x$ is the aircraft taxiing distance. The first moment and secondary moment of runway unevenness are acquired with the equation(13), from which it is known that stochastic process of runway unevenness with the harmony superposition method is a stationary Gaussian random process with zero mean ${ }^{[12]}$.

Form the equation (13), the computer can simulate a runway unevenness track. In order to obtain the random response in the model, we have to simulate a large number of runway unevenness track samples, with a lot of response samples by solving the equations, and then get the statistics data of response. According to literature ${ }^{[13]}$, the statistical error of the mean of simulated runway unevenness track declines as the rate of $1 / \sqrt{S}$. The greater the number of samples is, the smaller statistical error is. However, the more accurate simulation results are, the longer the computer simulation time is. Therefore, we must determine samples which have small calculation error and shorter number. In the simulation, the confidence interval width of runway unevenness and the computer CPU time are calculated, which are shown in Fig 2, where the number of samples is 10000. 


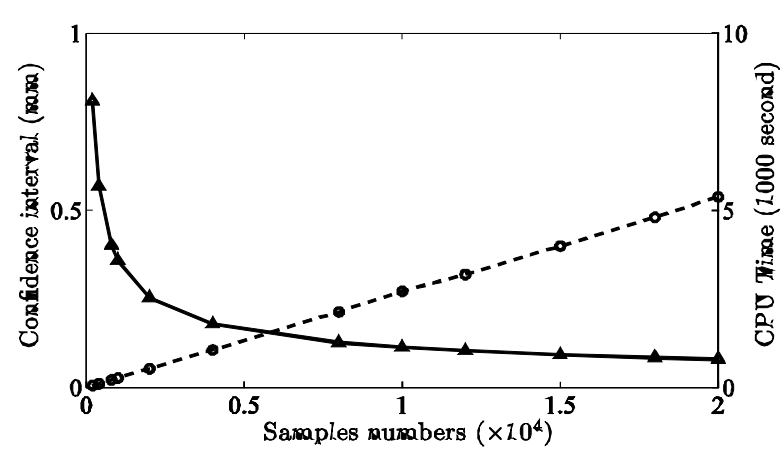

Fig.2 Determining the sample numbers, the solid line represents the confidence interval width and the imaginary line represents the computer CPU time.

\section{Numerical simulation and verification}

The secondary road surface spectrum at the airport is selected, which the parameter A and C of equation (9) are determined. The parameters of runway unevenness are shown in the Tab 1. According to the equation (13), a track of runway unevenness can be acquired with the number of 10001, which the space step size is $0.07 \mathrm{~m}$. The number of track of runway unevenness is $S$. At the $95 \%$ confidence level, the runway unevenness mean of the tracks is shown in Fig 3.

\begin{tabular}{ccc} 
& Tab. 1 Simulated parameters \\
\hline$A=2$ & $\Delta x=0.07 \mathrm{~m}$ \\
$C=0.0242$ & $N=200$ \\
$V=70 \mathrm{~m} / \mathrm{s}$ & $f_{1}=0.5 \mathrm{~Hz}$ \\
$X=700 \mathrm{~m}$ & $f_{2}=35 \mathrm{~Hz}$ \\
\hline
\end{tabular}

The autocorrelation function of runway unevenness can be acquired from the equation (14) ${ }^{[14]}$,

$$
R_{q q}\left(x_{r}\right)=\frac{1}{S X} \int_{0}^{X} \sum_{k=1}^{S} q_{k}(x) q_{k}\left(x+x_{r}\right) d x
$$

where, $R_{q q}\left(x_{r}\right)$ is the autocorrelation function of runway unevenness, $x_{r}$ is the interval distance between data points of runway unevenness, $q_{k}(x)$ is the $k$ track of runway unevenness. The curve is shown in the Fig 4, from which we can see that the curve of simulation autocorrelation function approximates to the curve of the theoretical autocorrelation function. With the larger $x_{r}$, the simulation autocorrelation function is closer to the square mean of theoretical autocorrelation function ${ }^{[15]}$.

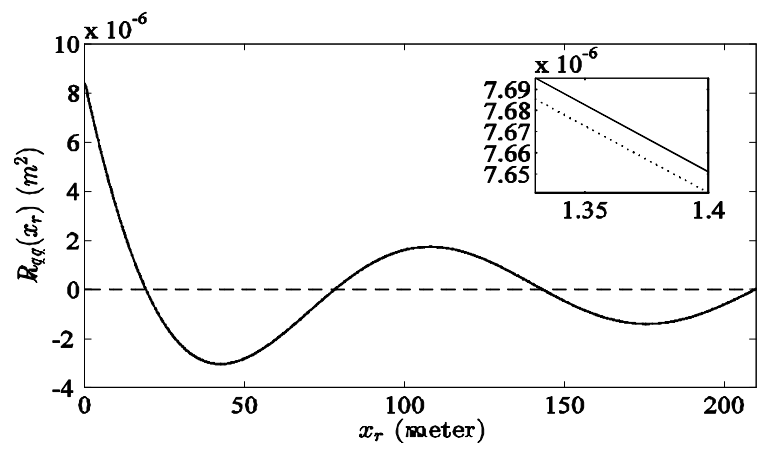

Fig. 4 The solid line represents the theoretical auto-correlation function, the dotted line represents the simulated auto-correlation function and the imaginary line represents the theoretical mean.

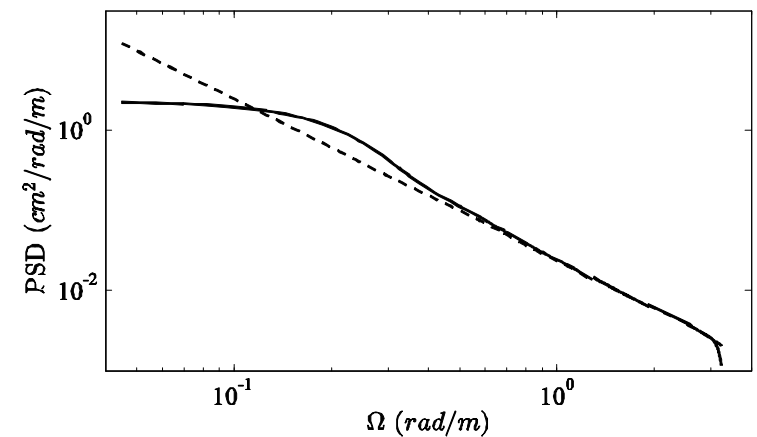

Fig.5 Fitting of power spectral density, the solid line represents the theoretical PSD of unevenness and the imaginary line represents the simulated data's spectrum.

From the literature ${ }^{[16]}$, the power spectral density can be achieved, shown in the Fig 5 , from which we can see that the simulation power spectral density is close to the theoretical power spectral density. It is shown that the simulation runway unevenness data is reliable. 


\section{The numerical simulation and statistical analysis of random response}

\section{The statistical magnitude of system response}

According to the literature ${ }^{[17]}$, the expressions of the mean of distance, the mean square value of the mean and the mean of autocorrelation function are described as follows,

$$
\begin{array}{r}
m_{y_{1}}(t)=E\left[y_{1}(t)\right]=\frac{1}{S} \sum_{k=1}^{S} y_{1_{k}}(t) \\
\sigma_{y_{1}}{ }^{2}(t)=E\left[y_{1}^{2}(t)\right]=\frac{1}{S} \sum_{k=1}^{S} y_{1_{k}}{ }^{2}(t) \\
R_{y_{1} y_{1}}\left(t_{r}\right)=\frac{1}{S T} \int_{0}^{T} \sum_{k=1}^{S} y_{1_{k}}(t) y_{1_{k}}\left(t+t_{r}\right) d t
\end{array}
$$

Tab.2 Initial conditions

where, $y_{1_{k}}(t)$ is the system response of track $k,{y_{1}}^{2}(t)$ is the square of system response of track $k . T=X / v$, which is the whole time. $t_{r}=x_{r} / v$, which is the time interval. The initial conditions are shown in the Tab 2 and the landing gear model parameters are shown in the Tab 3.

Tab.3 Landing gear model parameters

\begin{tabular}{cc}
\hline$P_{0}=1.6 \times 10^{6} \mathrm{~Pa}$ & $A_{0}=6.412 \times 10^{-4} \mathrm{~m}^{2}$ \\
$A=1.376 \times 10^{-2} \mathrm{~m}^{2}$ & $k_{t}=1.5 \times 10^{6} \mathrm{~N} / \mathrm{m}$ \\
$\varsigma=0.3$ & $c_{t}=2.6 \times 10^{4} \mathrm{~N} . \mathrm{s} / \mathrm{m}$ \\
$V_{0}=6.88 \times 10^{-3} \mathrm{~m}^{3}$ & $g=9.81 \mathrm{~m} / \mathrm{s}^{2}$ \\
$\rho=912 \mathrm{~kg} / \mathrm{m}^{3}$ & $k_{m}=7 \times 10^{3} \mathrm{~N} . \mathrm{s} / \mathrm{m}$ \\
$m_{1}=4832.7 \mathrm{~kg}$ & $k_{n}=1 \times 10^{4} \mathrm{~N} . \mathrm{s}^{2} / \mathrm{m}^{2}$ \\
$m_{2}=145.1 \mathrm{~kg}$ & $\gamma=1.865$ \\
\hline
\end{tabular}

\section{Confidence interval}

According to the central-limit theorem, no matter what distribution variable $u$ submits, as long as the sampling capacity $M$ is enough, the sampling distribution of subsample mean is close to the Gaussian distribution. If the subsample variance is unknown, it is close to the T distribution.

$$
\bar{u}=\frac{1}{M} \sum_{j=1}^{M} u_{j}
$$

$$
\sigma_{U}^{2}=\frac{1}{M-1} \sum_{j=1}^{M}\left(u_{j}-\bar{u}\right)^{2}
$$

where, $u_{j}$ is the value of random variable $u$ with serial number $j$. When the variance is unknown, the expression is given as follows, $\mu$ is the real mean of $u$. At $95 \%$ confidence level, the confidence of $\mu$ is as follows.

$$
\frac{u-\mu}{\sigma_{U} / M} \sim t(M-1)
$$

$$
\left[\bar{u}-\frac{\sigma_{U} t_{M-1 ; \alpha / 2}}{\sqrt{M}}, \bar{u}+\frac{\sigma_{U} t_{M-1 ; \alpha / 2}}{\sqrt{M}}\right]
$$

\section{The analysis result of statistical magnitude}

All the statistical magnitude is based on the displacement $\left(y_{1}\right)$. The curves of the mean of distance, the mean square value of the mean and the mean of autocorrelation function are shown in Fig6-Fig9

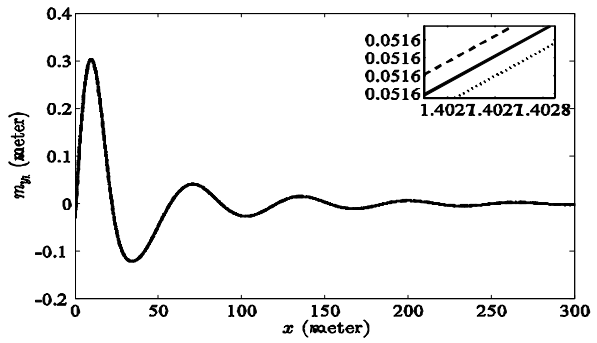

Fig.6 $y_{1}$ mean at $95 \%$ confidence level when taxiing at $v=70 \mathrm{~m} / \mathrm{s}$, the solid line represents $y_{1}$ mean, the imaginary line represents confidence upper limit and the dotted line represents confidence lower limit. After $300 \mathrm{~m}$, the mean is close to 0 .

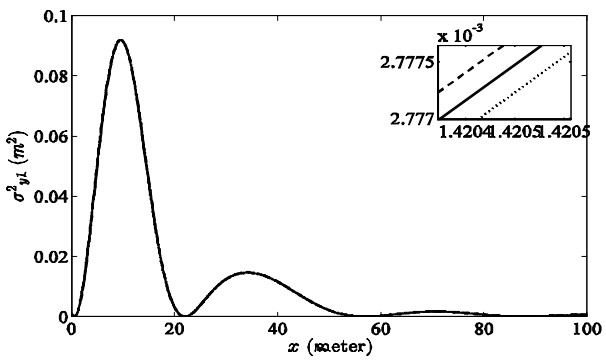

Fig.7 $y_{1}$ mean square value at $95 \%$ confidence level when taxiing at $v=70 \mathrm{~m} / \mathrm{s}$, the solid line represents the mean of $y_{1}$ mean square, the imaginary line represents confidence upper limit and the dotted line represents confidence lower limit. After $100 m$, the mean square is close to 0 . 


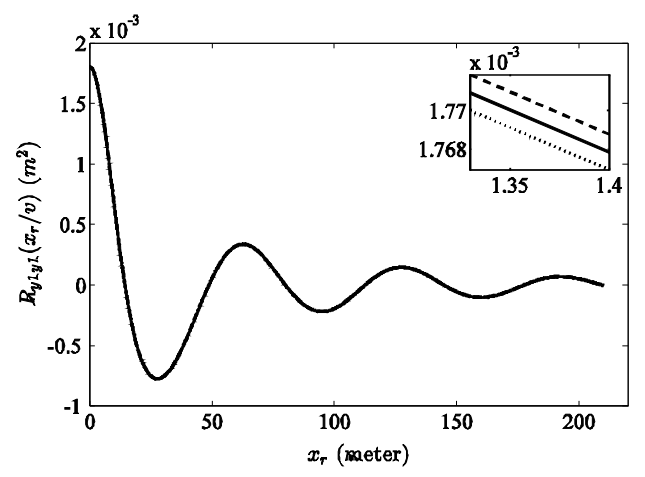

Fig.8 Mean of $y_{1}$ autocorrelation at $95 \%$ confidence level when taxiing at $v=70 \mathrm{~m} / \mathrm{s}$, the solid line represents the mean of $y_{1}$ correlation function, the imaginary line represents

confidence upper limit and the dotted line represents confidence lower limit.

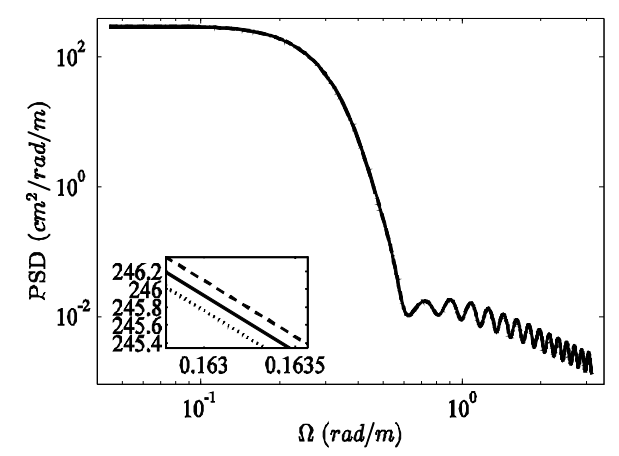

Fig.9 Mean of $y_{1}$ power spectral density at $95 \%$ confidence level when taxiing at $v=70 \mathrm{~m} / \mathrm{s}$, the solid line represents mean of displacement's PSD, the imaginary line represents the upper limit of mean and the dotted line represents the lower limit of mean.

The probability density curves of displacement can be acquired with the method of Scott. In this paper, we choose $x=21 \mathrm{~m}, x=100 \mathrm{~m}, x=200 \mathrm{~m}$ as three different position, the corresponding probability density curves can be obtained. By Carl square test, we can see that the displacement distribution is not Gaussian distribution which is shown in Fig10.

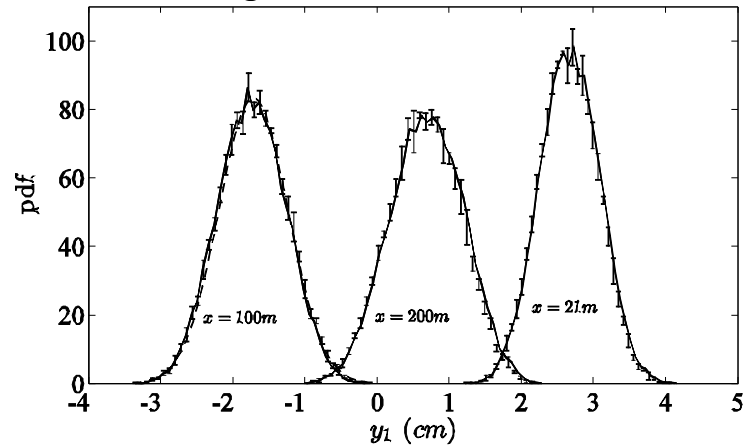

Fig.10 $y_{1}$ probability density mean of different distances at $95 \%$ confidence level when taxiing at $v=70 \mathrm{~m} / \mathrm{s}$, and the results of fitting test in Carl square are $\chi^{2}=105.97>\chi_{n, \alpha}^{2}=97.35 \chi^{2}=115.20>\chi_{n, \alpha}^{2}=100.75 \quad \chi^{2}=177.03>\chi_{n, \alpha}^{2}=101.88$

\section{Conclusion}

(1) Through computer simulation, we can determined the number of samples which have smaller confidence interval width unevenness and shorter the computer calculation time. It is shown that 10000 samples are sufficient, by the comparison between the analytical solutions and numerical solutions.

(2) Through the analysis of runway unevenness, the curves of autocorrelation function and power spectral density function were obtained. The results showed that the simulation track of the runway unevenness is Gaussian random process;

(3) A large number of displacement response samples are acquired through solving the non-linear dynamic equation with the input of random runway unevenness. At $95 \%$ confidence level, we can obtain the curves of statistical magnitudes which are the mean of distance, the mean square value of the mean and the mean of autocorrelation function.

(4)Through the probability density analysis of the displacement, the system response is non-Gaussian distribution with Gaussian excitation. The statistical magnitudes of displacement provide a good foundation for studying the fatigue loads and optimum control of landing gear.

\section{References}

[1] Li Si-zheng.Analysis of aircraft taxiing-induced response with multiple wheels and multiple landing gears[D].Xian:Northwestern Polytechnical University,2006. 
[2] Tung, C.C. The effects of runway roughness on the dynamic response of airplanes[J]. Journal of Sound and Vibration, 1967,5(1): 164-172.

[3] Kirk, C.L.,P.J. Perry.Analysis of taxiing induced vibrations in aircraft by the power spectral density method[J]. Aeronautical Journal, 1971,75(723): 182-194.

[4] Liu Li,Yang Guo-zhu,He Qing-zhi.Influences of landing gear system on dynamic response in aircraft during taxiing[J]. Acta Aeronautica et Astronautica Sinica,1992,(06):266-273.

[5] Nie Hong,W.Kortuüm.Analysis for aircraft taxiing at variable velocity on unevenness runway by the power spectral density method $[\mathrm{J}]$. Transactions of Nanjing University of Aeronautics and Astronau, 2000,(01): 64-70.

[6] Liu Rui-chen.The analysis for dynamic response during airplane taxiing. Acta Aeronautica et Astronautica Sinica, 1987,(13):601-610.

[7] Cao Rong-sheng.Establishment of airplane landing gear model and simulation analysis of landing behavior.Harbin:Harbin Engineering University,2007.

[8] Wang, H,et al. An investigation of an active landing gear system to reduce aircraft vibrations caused by landing impacts and runway excitations[J]. Journal of Sound and Vibration, 2008, 317(1-2):50-66.

[9] Walls J H, Houbolt J C, Press H. Some measurements and power spectra of runway roughness[M]. National Advisory Committee for Aeronautics, 1954.

[10] Thompson W E. Measurements and power spectra of runway roughness at airports in countries of the North Atlantic Treaty Organization[J], 1958.

[11]Zhang Yong-lin.Time domain model of road irregularities simulated using the harmony superpositon method[J].Transactions of the CSAE, 2003,(06):32-35.

[12] Shinozuka,M.,G.Deodatis. Simulation of stochastic processes by spectral representation[J]. Applied Mechanics Reviews, 1991.,44(4):191-204.

[13]Zhu Wei-qiu.Random vibration[M].Beijing:Science Press,1992.382-384.

[14]Liu Rui-chen,Su Kai-xin,Shao Yong-qi.Aircraft landing gear strengh design guide[M].Chengdu:Sichuan Science and Technology Press,1989.698-705.

[15]Chang Jian-ping,Li Hai-lin.Random signals analysis[M].Beijing:Science Press,2006.57-58.

[16]Zhang De-feng.Analysis of probability and mathematical statistics in Matlab[M].Beijing:Machinery Industry Press,2010.176-180.

[17] Wei Zong-shu.Probability theory and mathematical statistics[M].Beijing:Higher Education Press,2008.231-232. 